\title{
ELECTRON BEAM INJECTION, DEFLECTION, AND TRANSPORT IN THE UNM BETATRON
}

\author{
E. Schamiloglu, D.M. Siergiej, and S. Humphries, Jr. \\ Department of Electrical and Computer Engineering \\ University of New Mexico \\ Albuquerque, New Mexico 87131
}

\begin{abstract}
We present results from recent experiments on the generation of high-power relativistic electron beams using an upgraded injector and the subsequent injection and transport of these beams in the UNM Betatron. The experiments described were performed with peak beam energies of about $420 \mathrm{keV}$. The electron beams were deflected onto the main axis of the accelerating ring using a transverse electrostatic field. The dependence of transported beam current on both the vertical and focusing magnetic field strengths is discussed.

\section{INJECTOR UPGRADE}

The electron beam injector, described in detail elsewhere [1], has been upgraded to provide voltages up to $600 \mathrm{kV}$ to a felt cathode electron beam diode, double the original peak voltage. In addition, a novel crowbar utilizing high voltage cable for a synchronized delay has replaced the saturable ferrite core switch. The transit tine of the voltage pulse on the high voltage cable determines the output injector pulse duration. The present cable length is set to provide a voltage pulse of $80 \mathrm{~ns}$. A right-angle bend in the cathode stalk leading to the diode, incorporated recently in the original injector [2], was preserved in the upgrade. A typical output pulse for a 420 $k V$ injector charge voltage is presented in Fig. 1. Note that the voltage varies by less than $4 \%$ during the $80 \mathrm{~ns}$ pulse duration. This injector upgrade was motivated by our desire to increase the electron trapping efficiency in the accelerating ring by reducing the spread in injected electron energy distribution. Greater than 500 shots have been taken with the upgraded injector with no degradation in output performance.
\end{abstract}

\section{BEAM DEFLECTION INTO ACCELERATING RING}

The electron beam, upon passing through a transparent anode mesh, is transported $36 \mathrm{~cm}$ to the electrostatic deflector using a straight solenoidal magnetic field of $0.10 \mathrm{~T}$ for focusing. $\mathrm{A}$ beam current of about $150 \mathrm{~A}$ is measured at the exit of the transport tube. This represents a transport efficiency of over $90 \%$ through the solenoid. A time-integrated photograph of Cherenkov light output from an emittance diagnostic (similar

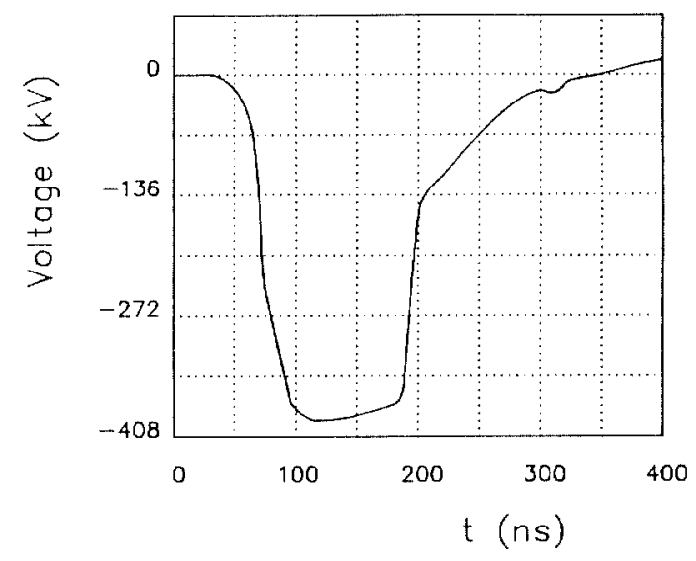

Fig. 1. Diode voltage for $420 \mathrm{kV}$ charge with apgraded injector. to the diagnostic described in Ref. 1) indicated that the beam had a divergent halo at the exit of the transport tube.

Upon exiting the transport tuhe, the heam encounters the combined forces of the transverse electrostatic field and reversing solenoidal lens field in the inflector chamber. The beam enters the chamber at an angle of $20^{\circ}$ and at an initial displacement from the main axis of $4 \mathrm{~cm}$. A diagram of the inflector chamber is shown in Fig. 2. The inflector is capable of providing a transverse electric field as large as $1.2 \mathrm{MV} / \mathrm{m}$.

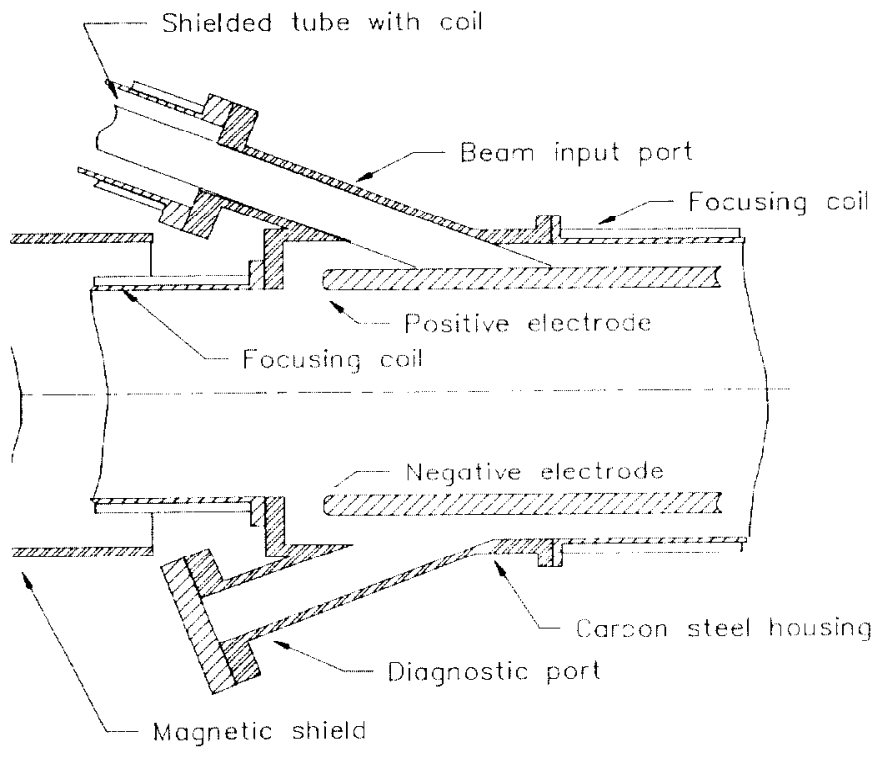

Fig. 2. Diagram of the inflector chamber.

(A detailed description of the inflector and its pulsed power circuit is given in Ref. 3.)

Beam transport in the inflector chamber is monitored by a diagnostics package consisting of a Rogowski coil and a graphite-coated disk of Pilot-B scintillator material. Figure 3 shows the experimental set-up for these studies. The timeintegrated bean profile is obtained by photographing the Cherenkov light emission from the scintillator. The observed horizontal and vertical displacements of the beam centroid as a function of the transverse electric field was compared with a computer calculation of single particle orbits in the combined fields [4]. Figures 4 and 5 present a comparison between beam displacement measurements and predictions. The data points were taken with a peak focusing field of about $0.023 \mathrm{~T}$. For $400 \mathrm{keV}$ electrons, this value of focusing field is predicted to result in an equilibrium beam radius of $2 \mathrm{~cm}$ (neglecting forces due to beam emittance). Our observation was that the beam radius was significantly larger than the equilibrium value. The discrepancy between the observed and predicted displacements is probably the result of beam emittance and possible mismatch. The large error bars on the experimental data points reflect the uncertainty in measuring the beam centroid 
when the profiles are very broad, as was the case in these runs with a focusing field of $0.023 \mathrm{~T}$. It was observed that when the focusing field strength was increased, the beam distribution was better defined and the displacements were in good agreement with the predictions. However, those beams were not well centered on the main axis of the confinement ring and therefore could not enter the first curved section of the accelerating ring.

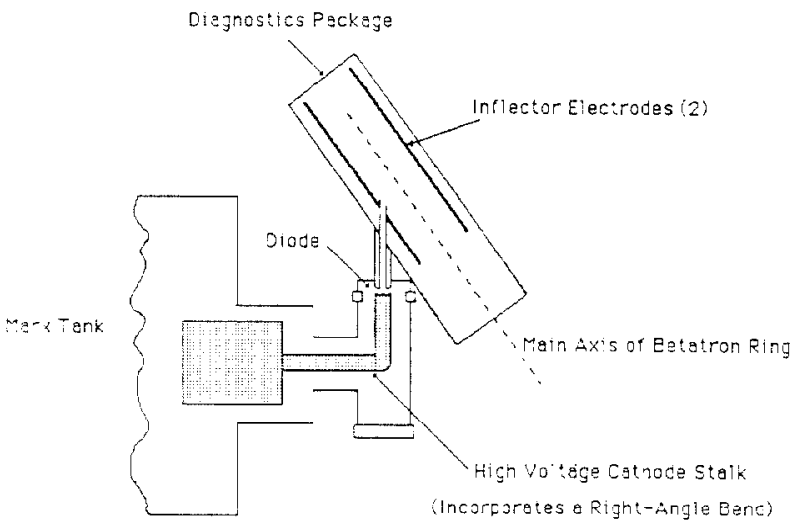

Fig. 3. Schematic of diode and inflector chamber.

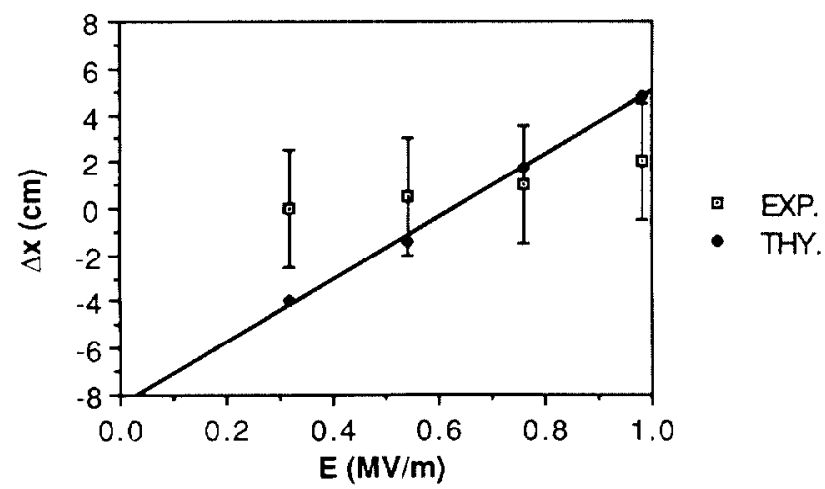

Fig. 4. Horizontal beam displacement in inflector chamber as a function of transverse electric field $\left(B_{O}=0.023 \mathrm{~T}\right)$.

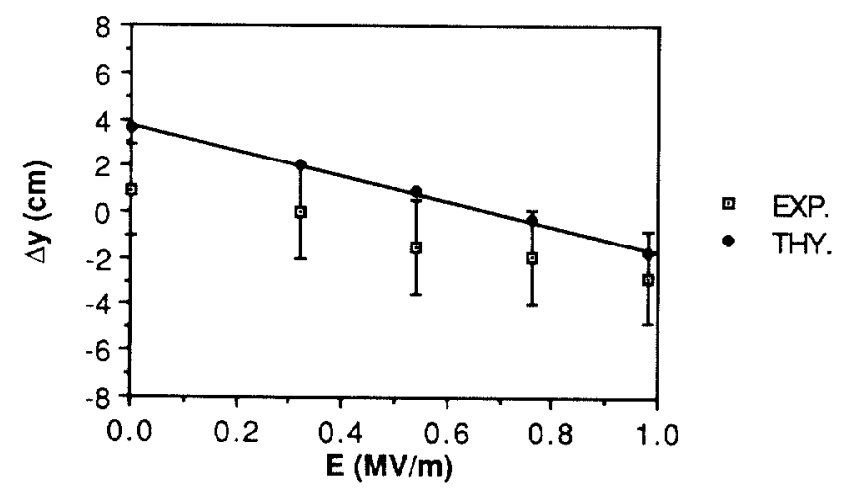

Fig. 5. Vertical beam displacement in inflector chamber as a function of transverse electric field $\left(B_{O}=0.023 T\right)$.

\section{BEAM TRANSPORT IN THE ACCELERATING RING}

In order to study the transport of the clectron beam around the entire accelerating ring, the diagnostics package at the end of the inflector chamber was removed and the inflector assembly was connected to the ring. The diagnostics used for the transport studies were three Rogowski coils, one located in the first accelerating gap at the entrance to the first $180^{\circ}$ curved section (CMON 1), one located at the exit of the first curved section (CMON 2), and one located in the second accelerating gap at the entrance to the second $180^{\circ}$ curved section (CMON 3). In addition, a beam centroid monitor consisting of a pair of balanced magnetic probes was installed in the middle of the first curved section. This monitor was oriented to observe the horizontal displacement of the beam during its transport in the curved section.

We begin our discussion of beam transport in the accelerating ring by referring to Fig. 6 where we present measurements of the beam current at three locations around the ring as a function of peak focusing field. A deflector field of $0.5 \mathrm{MV} / \mathrm{m}$ and a vertical field of $2.4 \mathrm{mT}$ were fixed in these studies. From figures 4 and 5 we see that this value of deflector field resulted in a well-centered beam in the inflector chamber. In Fig. 6, the first current monitor, CMON 1, measures on the order of 90 A leaving the inflector chamber and entering the first curved section of the ring. Only about 30 $A$ is transported around the first curved section. A peak focusing field of about $0.023 \mathrm{~T}$ results in the maximum transported beam current. The losses in the first curved section probably result from a large fraction of the entering beam electrons having significant non-axial velocity components.

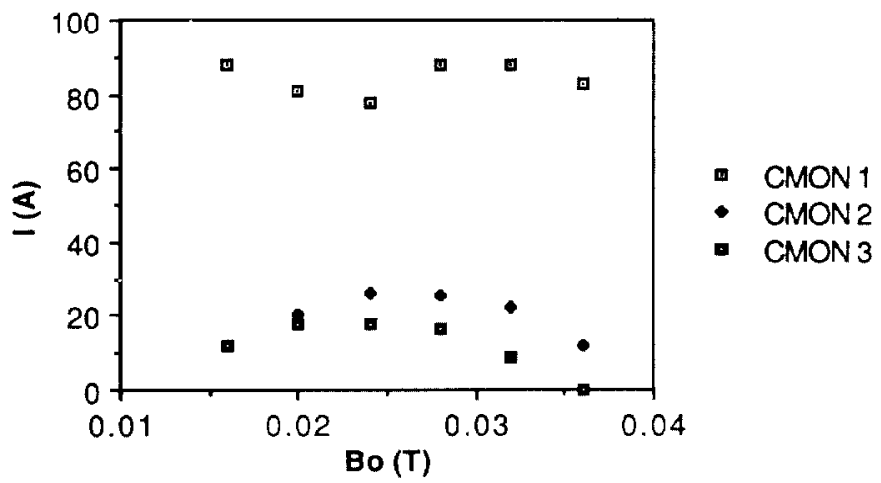

Fig. 6. Transported beam current as a function of peak focusing field $\left(E=0.5 \mathrm{MV} / \mathrm{m}, B_{v}=2.4 \mathrm{mT}\right)$.

The effect of the vertical field magnitude on transported beam current is evident in Fig. 7. On the left ordinate we plot the beam current measured at the exit of the first curved section and on the right ordinate we plot the horizontal beam displacement in the middle of the first curved section. A vertical field of about $2.5 \mathrm{mT}$ results in the maximum transported beam current. This vertical field is consistent with an electron Larmor radius of $1 \mathrm{~m}$ (for a $400 \mathrm{keV}$ electron), the radius of curvature of the accelerating ring. In addition, the larger vertical field strength resulted in the beam centroid being displaced towards the inner radius of the ring, as would be expected.

In summary, we have upgraded the electron beam injector to double the original peak voltage. The inclusion of a novel crowbar has resulted in an 80 ns pulse length with injector voltage being constant within $4 \%$. An electrostatic deflector is used to bend the beam onto the main axis of the accelerating ring. Transport studies have indicated that about $30 \mathrm{~A}$ of current is measured after the first curved section of the ring, but that an appreciable number of electrons are lost in 
propagating around the system. Optimizing the matching of the beam leaving the transport tube with the focusing field of the accelerating ring should allow for improvoments in beam transport efficiency.

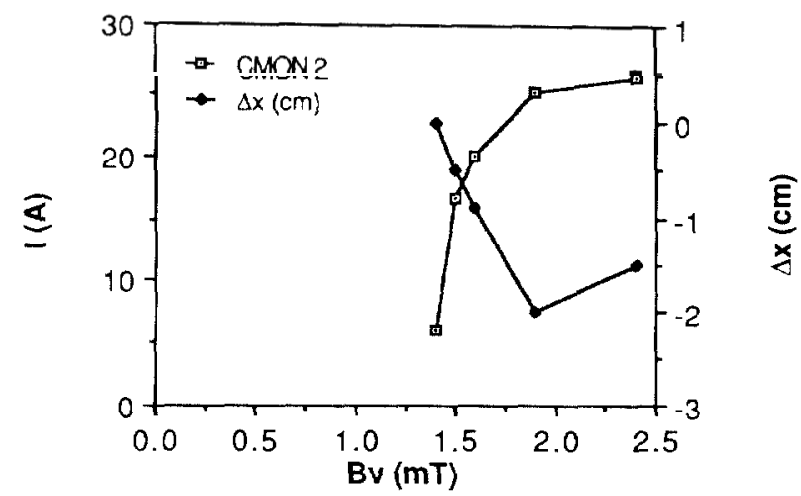

Fig. 7. Transported beam current and horizontal beam displacement as a function of vertical field $(E=0.5 \mathrm{MV} / \mathrm{m}$, $B_{0}=0.023 T$ ).

\section{ACKNOWLEDGMENTS}

The authors would like to thank R.L. Terry and A. Fratenglass for their technical assistance. This work was supported by the U.S. Office of Naval Rescarch under Contract No. N00014-84K-024,

\section{REFERENCES}

1. S. Humphries, Jr., L.K. Len, and C.B. Allen, Rev. Sci. Instr. 58, $796(1987)$.

2. E. Schamiloglu, R.L. Terry, and S. Humphries, Jr., Bull. Am. Phys. Soc. 33, 1984 (1988).

3. S. Humphries, Jr., University of New Mexico Betatron Project Technical Note 2 (1985).

4. S. Humphries, Jr., University of New Mexico Betatron Project Technical Note 11 (1988). 